\title{
Chapter 1 \\ Flexible and Dynamic Use of Spectrum: the Cognitive Radio Approach
}

\author{
E. Del Re, G. Gorni, L. S. Ronga and R. Suffritti
}

\begin{abstract}
The globalization of mobile and wireless communications for Next Generation Networks will require an efficient use of the scarce resource of radio spectrum. A cognitive radio approach is considered as a promising and suitable solution to solve this problem. We will address the cognitive radio approach by two viewpoints: heuristics and game theory. In particular the latter one, described in details, can provisionally represents an efficient solution.
\end{abstract}

E. Del Re, G. Gorni, L. S. Ronga and R. Suffritti

CNIT - University of Florence

e-mail: enrico.delre@unifi.it

e-mail: gherardo.gorni@unifi.it

e-mail: luca.ronga@cnit.it

e-mail: rosalba.suffritti@cnit.it 



\section{Contents}

1 Flexible and Dynamic Use of Spectrum: the Cognitive Radio

Approach ................................. 1

E. Del Re, G. Gorni, L. S. Ronga and R. Suffritti

$1.1 \quad$ Introduction ................................. 4

1.2 Challenges in Next Generation Networks ............... 4

1.3 Cognitive radio: the last frontier of spectrum exploitation ....... 6

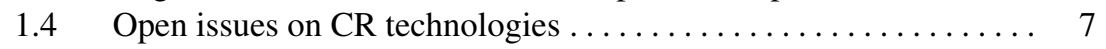

1.5 Heuristic Methods for Cognitive Radio ................ 8

1.5.1 Cognitive Radio Model..................... 8

1.5.2 Application Scenarios ....................... 12

1.5.3 Performance............................ 14

1.6 How Game Theory can help cognitive radio . ............. 18

1.6.1 Basics elements and different types of game ........ 20

1.6.2 Example of application to telecommunications ........ 22

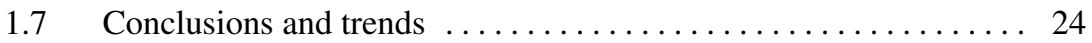

References .................................... 25 


\subsection{Introduction}

In the next future, the increasing and continuous demand of services anytime and anywhere and, therefore, the mandatory globalization of mobile and wireless communications for Next Generation Networks services will require a more and more efficient use of the, by now, scarce radio spectrum resources. The traditional communication systems which imply an a priori association of the frequency band, the service assigned to it and the used technology, need to become much more flexible, efficient and easy-to-use dynamic systems able to cope with the requirements and constraints of the environment and the users. A Cognitive Radio (CR) approach can be considered as a promising and suitable solution to solve this problem. The CR technologies are able to modify this current communication paradigm because they define a system able to sense the electromagnetic environment (spectrum sensing), detect the spectral resources actually occupied in a given temporal interval and in a given location, and use the free bands (holes) for its own communication.

In this work the Cognitive Radio idea, named nowadays also as the last frontier of the technology, will be presented as one of the most innovative technology which can help in the next development of new communication systems and it will be analyzed by two viewpoints: heuristic methods and Game Theory approach. The former approach deals with the coexistence problem of two systems, one licensed and one named "cognitive" terminal, which can share the radio spectrum resources if the cognitive device is able to use efficiently free spectrum holes, without interfering with the licensed communication service. In the latter one, instead, the Game Theory is considered as suitable mathematical instrument able to analyze, model and manage the contention of radio resources.

The chapter is organized as follows. In Section 1.2 the new challenges which the next generation of wireless systems will have to face, will be introduced. In Section 1.3 the Cognitive Radio concept will be described in more details while in Section 1.4 the Open issue in this field will be presented. In Section 1.5, the adopted cognitive strategy, based on an heuristic method, and the considered application scenario will be described and some obtained results presented. Section 1.6 will be focused on the Game Theory approach and on its potential application in telecommunication field. Some concluding remarks will be, finally, given in Section 1.7.

\subsection{Challenges in Next Generation Networks}

The increasing need of a global and interactive environment wherein users can access, more and more easily, to services and applications and the continuous demand for bandwidth in wireless communications generates serious warnings of spectrum saturation both in licensed and unlicensed bands. But the scarceness of spectrum resources is a real issue? Traditional spectrum policy is driven by the general concept of protection of relevant services from interference. The effect is that large portions of spectrum is locked on vast geographical areas for a very long time [1]. 
Although this approach has led to the development of many successful services (i.e. tv broadcasting, cellular telephony, wireless access to Internet) it also introduced a diffused inefficiency in large portions of the radio spectrum. As cited in [1], a recent report presenting statistics regarding spectrum utilization shows that even during the high demand period of a political convention such as the one held between August 31 and September 1, 2004 in New York City, only about 13 percent of the spectrum opportunities were utilized [2]. The real issue in the spectrum debate appears to be focused more on efficiency rather than exploration of new frequencies. This motivates the increasing interest in capacity exploiting technologies like MIMO, Ultra Wideband or Cognitive Radio. These technologies could enable for Next Generation Networks which aim at the convergence of digital contents (video, data, voice) towards IP-based networks and the diffusion of high-speed broadband access [3]. The Next Generation Networks will enable a number of key features that can be particularly beneficial to a wide array of potential services. While some of these services can be offered on existing platforms, others benefit from the advanced control, management, and signaling capabilities of NGNs. The most relevant services we are likely to use in the near future are strictly related to the features offered by NGNs. They are:

Voice Telephony: NGNs will likely need to support various existing voice telephony services (e.g., Call Waiting, Call Forwarding, 3-Way Calling).

Connectivity Services: Allows for the real-time establishment of connectivity between endpoints, along with various value-added features (e.g., bandwidth-ondemand, connection reliability/resilient Switched Virtual Connections [SVCs], and bandwidth management/call admission control).

Multimedia Services: Allows multiple parties to interact using voice, video, and/or data. This allows customers to converse with each other while displaying visual information. It also allows for collaborative computing and groupware.

Virtual Private Networks (VPNs): Voice VPNs improve the interlocation networking capabilities of businesses by allowing large, geographically dispersed organizations to combine their existing private networks with portions of the PSTN, thus providing subscribers with uniform dialing capabilities. Data VPNs provide added security and networking features that allow customers to use a shared IP network as a VPN.

Public Network Computing (PNC): Provides public network-based computing services for businesses and consumers.

Unified Messaging: Supports the delivery of voice mail, email, fax mail, and pages through common interfaces. Through such interfaces, users will access, as well as be notified of, various message types (voice mail, email, fax mail, etc.), independent of the means of access (i.e., wireline or mobile phone, computer, or wireless data device).

Information Brokering: Involves advertising, finding, and providing information to match consumers with providers.

E-Commerce: Allows consumers to purchase goods and services electronically over the network. 
Call Center Services: A subscriber could place a call to a call center agent by clicking on a Web page. The call could be routed to an appropriate agent, who could be located anywhere, even at home (i.e., virtual call centers).

Interactive gaming: Offers consumers a way to meet online and establish interactive gaming sessions (e.g., video games).

Distributed Virtual Reality: Refers to technologically generated representations of real world events, people, places, experiences, etc., in which the participants and providers of the virtual experience are physically distributed. These services require sophisticated coordination of multiple, diverse resources.

Home Manager: With the advent of in-home networking and intelligent appliances, these services could monitor and control home security systems, energy systems, home entertainment systems, and other home appliances.

All these services will be accessed both from fixed and mobile locations. In the latter case, innovations in wireless communications are essential. The path to next generation wireless communications is characterized by a few but challenging milestones. One of them is the full IP convergence of all services. Several driving protocols and technologies are ready for the complete abstraction of transport to IP (i.e. VoIP, IP QoS, broadcasting and conferencing services over IP) but several issues are still open in their integration. Another key element of future generation systems is the context awareness giving access to communications services which dependent from current user location and activity. VICOM project [6] collects many technologies in this area. The third milestone is the ubiquitous availability of large communications resources. This last step is obtained through consistent improvements in transmission and reception techniques, the use of Shannon-limit achieving channel coding, cooperative techniques, spectrum sharing and dynamic spectrum access (DSA) through the adoption of the Cognitive Radio approach. Finally, a fourth challenging milestone for NGNs can be identified in the huge number of wireless devices will be active in next decades. This aspect becomes critical considering that, in the 2020 time-frame, the estimated global population will be around 7 billion people communicating through more than 7 trillion wireless devices. In this futuristic, but likely, context it is absolutely necessary to think to a flexible technology able to guarantee the communication among this huge number of devices. The Cognitive Radio technology thanks to its flexibility and dynamism is one of the best candidate to solve this problem, allowing the wireless NGNs to work properly exploiting efficiently the scarce radio resources will be available in next years.

\subsection{Cognitive radio: the last frontier of spectrum exploitation}

The term Cognitive Radio (CR) was introduced in [7] with reference to a communication system able to observe and learn from the surrounding environment as well as to implement and adapt its own transmission modalities also to user requirements.

The concept of CR is originated from the contrast between an increasing demand of broadband services and the scarcity of radio resources. Recent studies of the FCC 
Spectrum Policy Task Force demonstrate that a large amount of licensed bands are under-utilized [8], i.e., a lot of spectral resources are reserved for specific services, but, actually, they remain unused for most of the time or unused in several locations. From these studies, the possibility of a $\mathrm{CR}$ is envisaged, i.e., a system able to sense the electro magnetic environment (spectrum sensing), detect the spectral resources actually occupied in a given temporal interval and in a given location, and use the free bands (holes) for its own communication. The search for available resources is not limited to spectrum portions dedicated to unlicensed communications, but is also extended to licensed bands. In this case, a CR system, called the secondary system, must coexist with a primary system, i.e., the license owner, without producing harmful interference, as shown in figure 1.2. Both earth and satellite systems can be considered for the role of primary and secondary users.

A CR system assumes that there is an underlying hardware and software system infrastructure that is able to support the flexibility demanded by the cognitive algorithms [9]. In this case, the abstraction of hardware capabilities for radio software architecture is a primary design issue because it is advisable to isolate the cognitive engine from the underlying hardware. In this context, the Software Defined Radio (SDR) represents the essential enabling technology for its characteristics. SDR is a methodology for the development of applications in a consistent and modular fashion such that both software and hardware components can be promptly reused for different implementations. SDR technology can also provide an advanced management of the available resources and facilitate the definition of interfaces for the coexistence of different communication services.

Considering what has been said before, a CR approach turns out to be one of the most promising technologies which can help to overcome the constraints of the current wireless systems in terms of efficient use of the spectrum resources and it can have, therefore, a key role in the development of a global access environment wherein there are more and more user devices and less and less free spectrum portions.

\subsection{Open issues on CR technologies}

The Cognitive Radio idea of exploiting the unused frequency bandwidths is really powerful and efficient by the point of view of flexibility in spectrum management. The use of licensed frequencies by secondary users in a transparent way to primary users is a challenging aspect for a wireless communications engineer. With transparent it is meant that the Cognitive Radios terminals do not impair licensed users communications. It is possible to address three issues in particular that make this challenge absolutely non-trivial.

In first place there is the matter of sensing and detecting primary licensed users. This is operation is mandatory for CR terminals since through that they can determine the presence or not of licensed users. Technically speaking this can be done estimating the noise temperature over a certain range of frequencies, determining 
eventual variations due to primary users. The issue is arising is not all primary users can be detected, but however they can be affected by interference due to secondary users transmission.

Another emerging issue is how the CR terminals access to spectral holes. Indeed, if a frequency band is found unoccupied after sensing, there will be strong interference if all secondary users try to access to the free resource in a straight way. Suited multiple access criteria must be defined in order to have the network working. Two different approaches can be pointed out to solve this issue: the first is given by heuristic methods which are more immediate to be applied even if not optimal, the second approach is given by game theory, which is a mathematical tool able to manage complex communications contexts. Both methods will be presented later on.

Assuming to have a channel access policy able to resolve the former issue, the third matter of interest has been found is about the amount of information required to realize a coordination between Cognitive Radios. Here a trade off has to be found between a complete information describing opportunely the environment of each $\mathrm{CR}$ terminal and a partial information which gives to CR terminal a less accurate description of the operating environment but it is produces less overhead as well.

\subsection{Heuristic Methods for Cognitive Radio}

This section shows the potential benefits of the adoption of a cognitive radio strategy, based on an heuristic method, to the coexistence problem of different services and systems on the same radio bands. The basic model of a Cognitive Radio (CR) system will be introduced and described as well as the assumptions introduced for the overall considered system. In this context, a CR system is defined by a secondary system which have to coexist with a primary system, which is the license owner, without producing harmful interference. In this study the problem is formalized as a constrained maximum search, where the objective function is the rate of the secondary system subject to the preservation of the primary system quality requirements.

\subsubsection{Cognitive Radio Model}

In the following, the model of the overall system, composed of a primary and of a secondary component, and the coexistence problem, i.e., the exploitation of radio resources unused by the primary system, are investigated.

All the considered systems are OFDM based. Several reasons induce to consider OFDM as the principal candidate for implementing the physical layer of a CR system, ranging from its ability to combat multipath fading to a relatively simple way to manage spectrum occupancy. 
Let $P_{R x}(k)$ and $P_{T x}(k)$ be the received and the transmitted power on the $k$ th carrier of the system, $k=0,1, \ldots, K-1$, with $K$ total number of carriers, $C(k)$ the instantaneous channel matrix relative to the $k$ th channel and $N_{x}$ the noise power. The index $x$ stands for either 1 (denoting the primary system) or 2 (the secondary system). The solution space is represented by the $P_{T 2}(k)$ power vector, obtained by the Cognitive Radio strategy. Fig.1.1 depicts schematically the CR system.

The adopted cognitive strategy is derived for a secondary system able to collect all

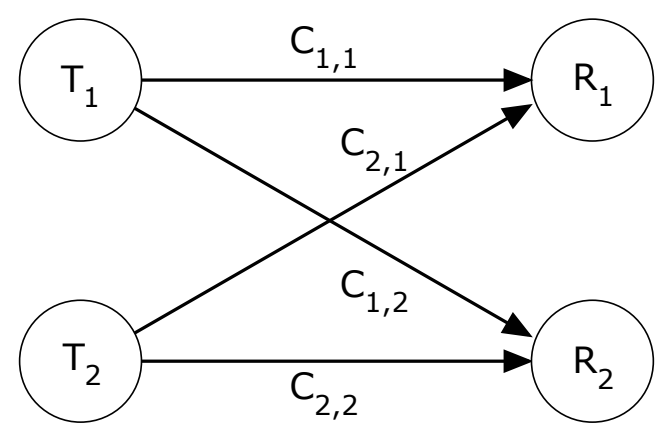

Fig. 1.1: Cognitive scenario

the relevant propagation information of both systems. The power vector transmitted by the primary system, the channel impulse response and the statistical measures of the thermal noise are supposed known by the secondary system. The considered system is regulated by (1.1) and (1.2), where $k$ is the subcarrier index.

$$
\begin{aligned}
& P_{R 1}(k)=C_{1,1}(k) P_{T 1}(k)+C_{2,1}(k) P_{T 2}(k)+N_{1}(k) \\
& P_{R 2}(k)=C_{2,2}(k) P_{T 2}(k)+C_{1,2}(k) P_{T 1}(k)+N_{2}(k)
\end{aligned}
$$

The problem can be formulated as a constrained multi-variable maximum search, where the objective function, namely $R$, is the secondary system bit-rate and the constraints are given by the minimum quality, in terms of maximum tolerable BER, that must be assured for the primary and the secondary system. The objective function and the constraints are given by: 


$$
\begin{aligned}
& \max _{b_{2}(k)} R=\sum_{k=0}^{K-1} b_{2}(k) \\
& \frac{P_{T 2}(k) C_{2,2}(k)}{N_{2}(k)+P_{T 1}(k) C_{1,2}(k)} \geq \operatorname{SINR}_{R 2, \min }\left(b_{2}(k)\right) \\
& \frac{P_{T 1}(k) C_{1,1}(k)}{N_{1}(k)+P_{T 2}(k) C_{2,1}(k)} \geq \operatorname{SINR}_{R 1, \min } \\
& \sum_{k=0}^{K-1} P_{T 2}(k) \leq P_{T 2, \text { tot }}
\end{aligned}
$$

In the (1.3), $b_{2}(k)$ indicates the value of the secondary bit-rate for the $k$ th carrier. This is equal to 0 if the considered carrier is not used by the secondary. Otherwise, it assumes a value depending on the used modulation. Constraints (1.4) and (1.5), instead, are derived according to the signal-to-noise-and-interference ratio $(S I N R)$ at the secondary and primary receiver, respectively. Let $S I N R_{R 1, \min }$ and $\operatorname{SINR}_{R 2, \min }\left(b_{2}(k)\right)$ be the minimum SINR values that allows a reliable communication, i.e., values that allow a given BER to be achieved, on a given carrier of the primary and secondary system, respectively. We assume that an adaptive modulation is used for the secondary system, so that the quantity $\operatorname{SINR}_{R 2, \min }\left(b_{2}(k)\right)$ is a function of the number of bits delivered by the $k$ th carrier, i.e., the modulation scheme used on the carrier (to be chosen from a given set). The primary receiver target quality is expressed by a given value of $S I N R_{R 1, \text { min }}$, which results independent of $b_{2}(k)$ (a QPSK modulation is assumed to be used by the primary system). During the iterative allocation process the simpler modulation is considered first (e.g., QPSK) to compute $S I N R_{R 2, \min }$, and then, whenever possible, a modulation upgrade is performed recursively (see the power allocation algorithm described below). According to the constraints (1.5) and (1.4), the power on the $k$ th carrier of the secondary system is upper and lower bounded by the following expressions:

$$
\begin{gathered}
P_{T 2}(k) \leq \frac{P_{T 1}(k) C_{1,1}(k)-N_{1}(k) \operatorname{SINR}_{R 1, \min }}{\operatorname{SINR}_{R 1, \min } C_{2,1}(k)} \\
P_{T 2}(k) \geq \frac{\operatorname{SINR}_{R 2, \min }\left(P_{T 1}(k) C_{1,2}(k)+N_{2}(k)\right)}{C_{2,2}(k)}
\end{gathered}
$$

The constraint in (1.6) is the total amount of power transmitted by the secondary device. This is ruled by (1.6) and derived by regulatory limitations on the secondary transmission power.

The described constraints can sometime be contradictory: in this case the applied rule is to protect the primary service.

As concerns the quality constraint of the primary system, it is worth noting that for each subcarrier $k$ two possible cases may occur at the primary receiver. Let 
$\operatorname{SINR}_{R 1,2 \text { off }}$ be the signal-to-noise ratio experienced at the primary receiver before the secondary begins its transmission. The two possible cases are:

1. $\operatorname{SINR}_{R 1,2 o f f}(k)<\operatorname{SINR} R_{R 1, \min }$

2. $\operatorname{SINR}_{R 1,2 o f f}(k) \geq \operatorname{SINR} R_{R 1, \min }$.

In the former case, the primary system does not reach the desired quality level on the $k$ th carrier, so the primary does not use the carrier whereas the secondary system can fully exploit it. In the latter case, the primary system reaches the desired minimum SINR and eventually provides an useful margin to be exploited by the secondary.

The underlying idea of the proposed resource allocation strategy is the creation of a power allocation vector for the secondary allocation, iteratively updated until a stop condition is reached (i.e., when the total amount of secondary power is reached or a constraint is violated).

The allocation procedure is an iterative process where the instantaneous secondary resources at iteration $n$ are represented by two vectors, an integer vector $b^{(n)}$ and a real valued vector $P_{T 2}^{(n)}$, representing respectively the modulation and the allocated power for each subcarrier. The possible values of $b(k)^{(n)}$ are $\{0,2,4,6\}$ which identify an unallocated carrier, a QPSK, a 16-QAM and a 64-QAM symbol on the $k$ th carrier, in that order. An auxiliary vector $d^{(n)}$ represents the amount of power, at iteration $n$, necessary to increase the rate of the secondary system on each carrier. The rate increments are those that, if applied, change the corresponding value of $b(k)$ from 0 to 2 (activation of the carrier with a QPSK symbol), from 2 to 4 (modulation upgrade from QPSK to 16-QAM), from 4 to 6 (modulation upgrade from 16-QAM to 64-QAM). The algorithm is as follows:

Step 0: $n=0, b^{(0)}$ and $P_{T 2}^{(0)}$ are initialized to all-zero vectors of length $N$ (no carrier allocated to the secondary);

Step 1: $d^{(n)}$ is computed taking into account the constraints in (1.8) and (1.7), as well as the current allocation $b^{(n)}$ and $P_{T 2}^{(n)}$. The carriers where the lower power constraint is greater then the upper one are marked as 'unusable'.

Step 2: find the carrier for which a rate increment is possible with the lowest amount of secondary power, i.e. $\hat{k}=\arg \min _{k} d(k)^{(n)}$ (excluding the unusable carriers and those already allocated with the highest modulation order);

Step 3: if a valid $\hat{k}$ has been found, allocate the carrier by defining the modulation:

$$
b^{(n+1)}(k)= \begin{cases}b^{(n)}(k)+2 & \text { if } k=\hat{k}, \\ b^{(n)}(k) & \text { otherwise. }\end{cases}
$$

and the corresponding power: 


$$
P_{T 2}^{(n+1)}(k)= \begin{cases}P_{T 2}^{(n)}(k)+d(k)^{(n)} & \text { if } k=\hat{k}, \\ P_{T 2}^{(n)}(k) & \text { otherwise. }\end{cases}
$$

Step 4: increase the iteration index $n$

Step 5: compute the total amount of power allocated to the secondary; if $\sum_{k} P_{T 2}^{(n)}(k) \leq$ $P_{T 2, t o t}$ than go back to Step 1, otherwise use the allocation vectors $b^{(n)}$ and $P_{T 2}^{(n)}$.

It is worth noting that at each iteration there are two possible choices: allocate a new carrier to the secondary with a QPSK modulation or perform a modulation upgrade (e.g., QPSK to 16-QAM or 16-QAM to 64-QAM) on a carrier already allocated to the secondary service. The choices are equivalent in term of rate increment and it is selected the one with the minimum required power.

\subsubsection{Application Scenarios}

Cognitive Radio strategies are strictly related to their operating environment. In this section, two scenarios are taken into consideration: a fully terrestrial and a mixed satellite/terrestrial scenario. Fig. 1.2 depicts schematically the mixed terrestrialsatellite scenario. The considered scenarios have the objectives to investigate on the coexistence problem, following the model illustrated previously. A terrestrial infrastructure or a satellite system considered as primary systems, have to coexist with a mesh-based terrestrial telecommunication service. All the considered systems are OFDM based, but they exhibit different propagation conditions.

\subsubsection{Fully Terrestrial Context}

The first scenario is composed by a licensed primary system and a "cognitive" secondary system. Both are terrestrial systems and are characterized by a single transmitter and a single receiver. The two systems are not independent since they share the same radio resource. The signal from each transmitter represents a interfering component to the other system receiver. The secondary system operates with OFDM with the same carrier spacing as the primary one. It has, however, a more flexible power allocation scheme. Being cognitive, the operating parameters like frequency, modulation and power are modified by its software radio implementation. The possible modulations for the considered secondary system are QPSK, 16-QAM and 64QAM. With a constant bit error probability, the minimum required signal-to-noise ratio depends on the modulation order. The actual transmission mode is automatically selected by the secondary device based on the available sensed information. 


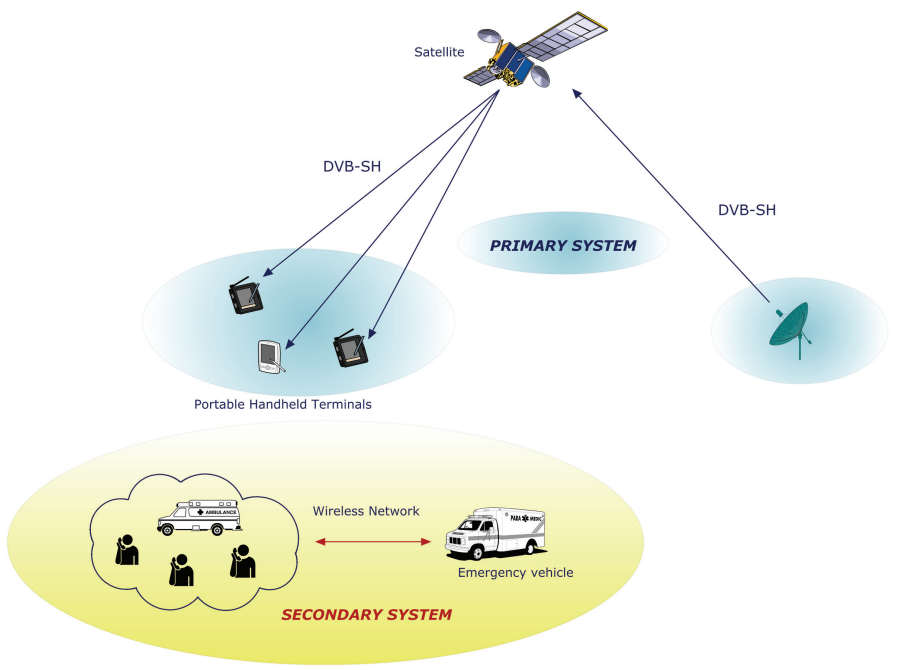

Fig. 1.2: Cognitive scenario for mixed terrestrial-satellite emergency communications.

\subsubsection{Channel Model}

The received signal is corrupted by different phenomena: a path loss term due to the transmitted distance calculated at the middle-band frequency, and a multi-path fading due to the propagation environment and terminal motion. The path loss is modeled by:

$$
L=10 \log _{10}\left(\frac{4 \pi d}{\lambda_{0}}\right)^{\alpha} d B
$$

where the exponent $\alpha$ models the attenuation dependence from the distance, $\lambda_{0}$ is the central frequency wave length and $d$ is the transmitter distance from the receiver. In the time-domain the channel exposes a finite impulse response of $L$ samples, resulting in a frequency selective channel response. A tapped-delay model with Rayleigh distributed coefficients has been adopted. The power delay profile is exponential as follows:

$$
\sigma_{n}^{2}=e^{-\beta n}
$$

where $\sigma_{n}^{2}$ is the variance of the $n$-th coefficient and $\beta$ is computed for a normalized mean power response. 


\subsubsection{Mixed Terrestrial/Satellite Context}

Differently from the case previously described, the second scenario is mixed, i.e. it is characterized by a licensed primary satellite system and a "cognitive" secondary terrestrial system. The primary system is a mobile satellite system based on DVBSH standard [17], while the secondary one is a terrestrial wireless meshed network that could be used in emergency situations. The two considered systems work in $\mathrm{L}$ band $(0.39-1.55 \mathrm{GHz})$ and exploit a context of coexistence in which the secondary one is allowed to take resources from another system without interfering in its normal operations.

\subsubsection{Channel Model}

The propagation channel for the considered mobile satellite channel at L-band is the Lutz (et others) model [18]. It is based on a two-state (GOOD-BAD) Markovchain for the fading process. According to this class of models, the amplitude of the fading envelope is divided into fast and slow fading. Slow fading events, normally due to large obstacles, are modelled as a finite state machine. Fast fading events, due to the irregularity of the obstacles (e.g. vegetative shadowing) and to the multipath propagation phenomenon can be additionally represented as superimposed random variations that follow a given probability density function (PDF) for each state. This channel, differently from the previous one, has a flat frequency response. Also in this case, the path loss term due to the transmitted distance calculated at the middleband frequency, has to be considered in the channel model.

\subsubsection{Performance}

In the following, the proposed CR strategy is validated through computer simulations for both considered scenarios. The terrestrial propagation exponent $(\alpha)$ is considered equal to 3.0 , valid for a medium density urban scenario while for the satellite scenario the value of $\alpha$ is assumed equal to 2.0. It is assumed that the number of carriers is $K=128$ for the fully terrestrial scenario and $K=853$ for the mixed terrestrial/satellite one.

\subsubsection{Fully Terrestrial Context}

The performance for the fully terrestrial scenario have been evaluated considering the achieved rate of the secondary system as the main performance index. Simulations have been conducted for six target $B E R$ values of the secondary system, from $10^{-3}$ to $10^{-8}$ and for three $E_{b} / N_{0}$ values for the primary receiver equal to $10 \mathrm{~dB}$, $15 \mathrm{~dB}$ and $20 \mathrm{~dB}$. 
The primary achieved rate depends only on the primary received $E_{b} / N_{0}$, since the cognitive radio strategy always preserve the primary rate.

The considered context has terminal displacements as shown in Fig. 1.3. Units are normalized to the primary distances.

The performance of the secondary system heavily depend on its target $B E R$ and

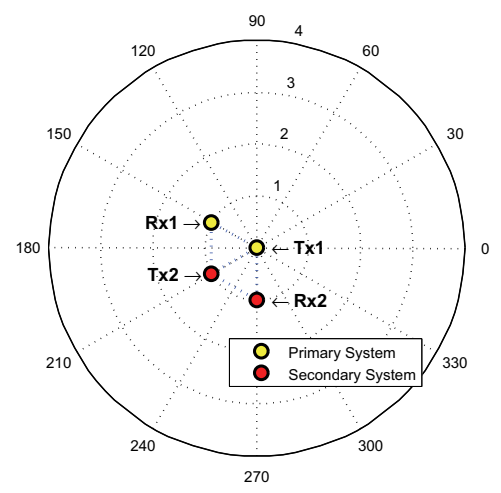

(a) Case $\mathrm{A}$

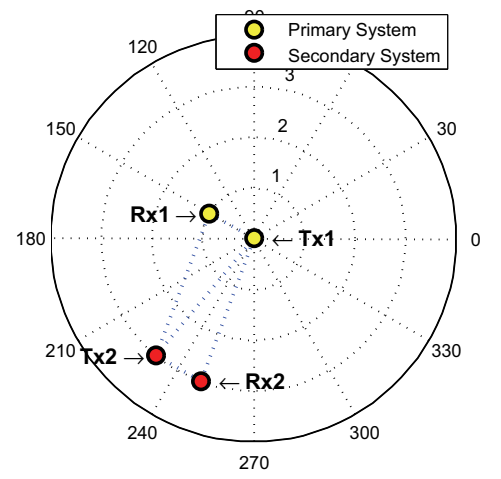

(b) Case B

Fig. 1.3: Fully terrestrial terminals displacements: polar rappresentation of the different distances considered

on the primary working point. In figure 1.4 and in figure 1.5 , for both considered displacements and each $E_{b} / N_{0}$ value of the primary, it is reported the $B E R$ values in log-scale function of the achieved secondary rate. In the first case (Case A), i.e. where the secondary terminals are further from the primary system, it is worth noting that, for a fixed $B E R$, the achieved rate increases as the primary $S N R$ increases. This is a well known limitation of Cognitive Radio systems, where low SNRs at the primary heavily impair the secondary achievable performances. In the second case (Case B), where the two systems are closer, it can be noted that the outcomes are different in comparison with the Case A. In fact, for a fixed $B E R$, the achieved rate increases as the primary $S N R$ decreases. This is due to the closeness between the two systems. In this case, the interference of the secondary is higher than in the Case A. The secondary can use only the carriers that the primary discards because of the fading and these increase as the primary $S N R$ decreases.

Another interesting element is represented by the modification of the experimented SINRs at the primary and secondary receivers before and after secondary transmission. Figure 1.6 reports for the Case A and for each subcarrier, the primary (left) and secondary (right) SINR (named SIR in the Figure) before and after secondary activation, for a fixed secondary $B E R$.

The considered primary $E_{b} / N_{0}$ values are 10,15 and $20 \mathrm{~dB}$, whereas the secondary $B E R$ is fixed to $10^{-3}$. The dashed line represents the SINR before the secondary activation. The channel frequency selectivity provides a variable response for both 


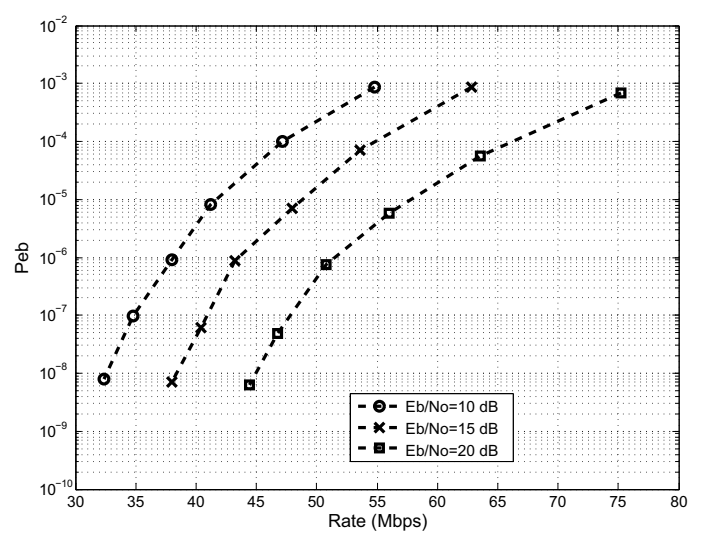

Fig. 1.4: BER vs. Rate for the secondary system: Case A

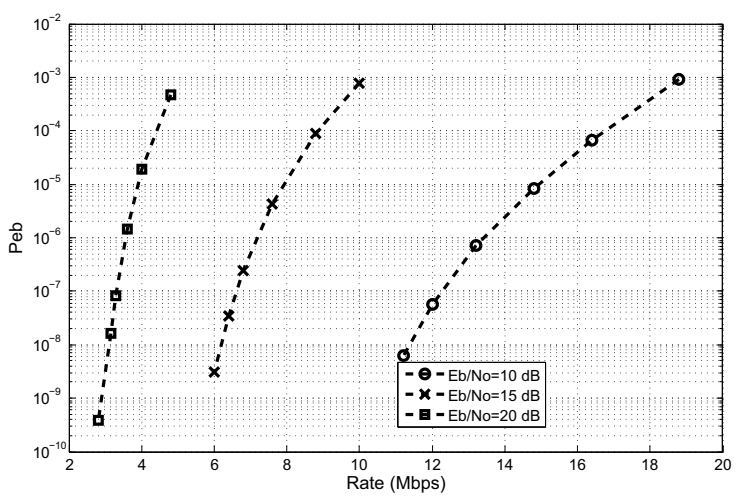

Fig. 1.5: BER vs. Rate for the secondary system: Case B

systems. The cognitive process places power where the secondary channel is in a good state, and after its activation, the corresponding primary SINR lowers (i.e. the interference from the secondary increases). As can be seen, the larger $E_{b} / N_{0}$, the larger the number of carriers where the secondary is allowed to allocate power; this explains the operating point dependence of Figure 1.4.

\subsubsection{Mixed Terrestrial/Satellite Context}

Also for the mixed terrestrial/satellite scenario, as the main performance index, the achieved rate of the secondary terrestrial system has been considered. In this case, the six target $B E R$ values of the secondary system are different with respect to the 

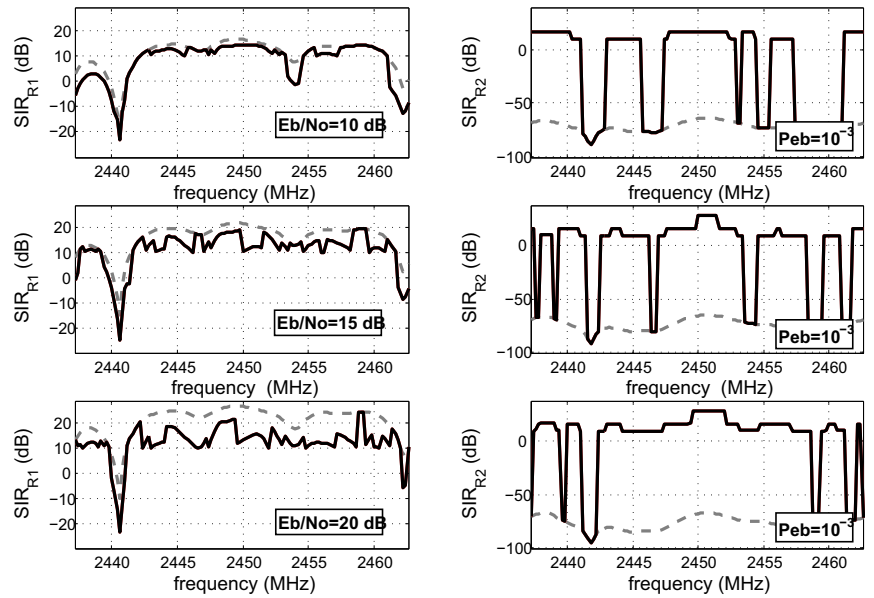

Fig. 1.6: SINR vs frequency for the primary (left) and secondary (right) systems: Case A

fully terrestrial scenario and they are: $10^{-2}, 5 \cdot 10^{-3}, 10^{-3}, 5 \cdot 10^{-4}, 10^{-4}, 5 \cdot 10^{-5}$; the three $E_{b} / N_{0}$ values for the primary receiver are, instead, the same of the previous scenario: $10 \mathrm{~dB}, 15 \mathrm{~dB}$ and $20 \mathrm{~dB}$. For this context, two different environments,

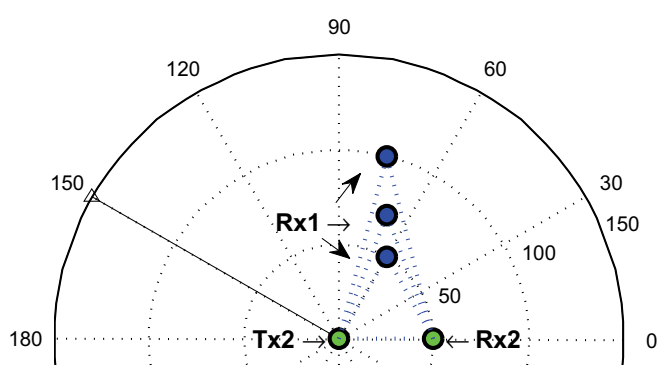

Fig. 1.7: Terminals displacements: polar rappresentation of the different distances considered

CITY and HIGHWAY, have been considered. The terminal displacements are shown in Figure 1.7. As it can be seen, the secondary terminals, $T x_{2}$ and $R x_{2}$, are fixed and their distance is set to 50.0 meters while the receiver of the primary system, $R x_{1}$, approaches the secondary cluster at different distances. The performance has been evaluated for three distance values among the three terminals: $100-100-50$ 
meters, $70-70-50$ meters and $25-25-50$ meters. In Figure 1.8, for each distance

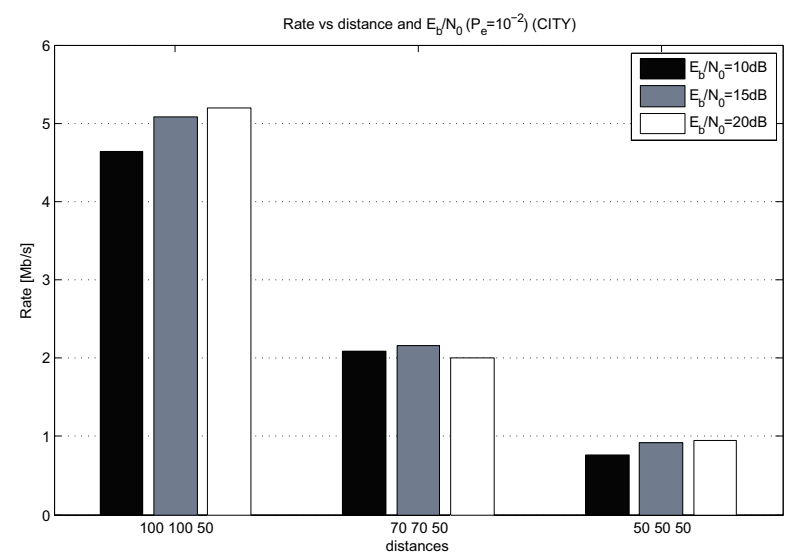

Fig. 1.8: Secondary Rate vs. Distances (CITY environment)

and for each $E_{b} / N_{0}$ value of the primary, it is reported the secondary rate value in the CITY environment with the target $B E R$ equal to $10^{-2}$. It is important to note that as the distances decreases, the secondary rate decreases as well. As already said, this is due to the increase of the interference between the two systems and to the lower power that the secondary can utilize when it is approaching to the primary in order to protect the licensed service.

Another interesting element is to consider the rate performance for the two analysed environments. In Figure 1.9, it can be seen that the achieved secondary rate is lower in the CITY case. This is due to the presence of a strong shadowing resulting in a impaired reception of the primary signal. The cognitive strategy has to guarantee the primary rate so the secondary has to minimize its transmission.

\subsection{How Game Theory can help cognitive radio}

Very often mathematics provide powerful instruments to deal with complex problems appearing unsolvable at first glance. In a Cognitive Radio operative environment such kind of situation can be found: a certain number of unoccupied frequency bandwidths, i.e. spectrum holes, potentially available for cognitive terminals. Assuming the existence of infrastructured communication system with centralized resource allocation for cognitive users, it would solve every arising problem in terms of occupation of spectrum holes. Actually the former solution it is not so convenient since an high number of CR terminals would imply a huge amount of data exchange with the system base station, with a consistent decrease of data throughput. Further- 


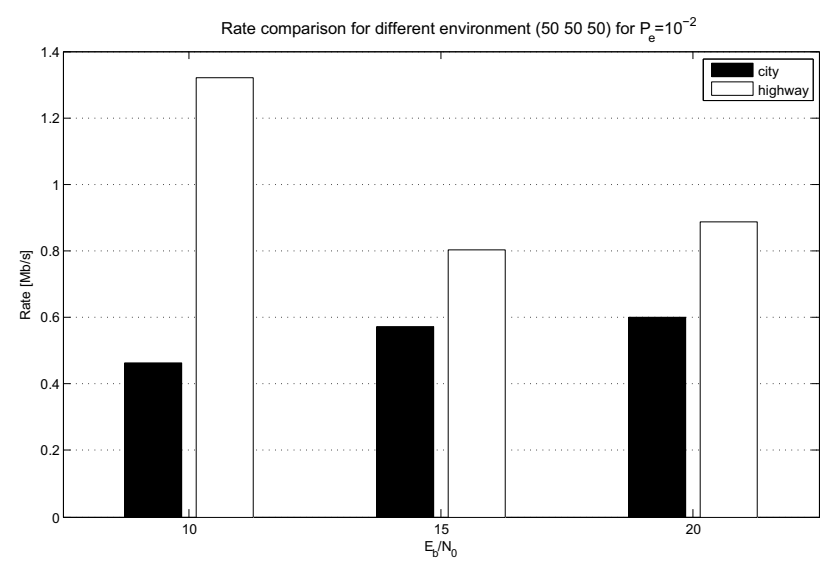

Fig. 1.9: Rate vs. $E_{b} / N_{0}$ for different environments

more each cognitive terminal senses an operative environment different from the other terminals one, e.g. a cognitive radio could detect several spectrum holes while a CR terminal nearby could detect just one available hole because it is surrounded by a lot of licensed users.

Then it is clear the need of approach the problem in a different way. Instead of an infrastructured system managing the radio resources as well, it could be imagined to make distributed the occupation of the spectrum holes. So there is the need of a tool able to analyze and model the contention of radio resources. Mathematics can help us with the former issue providing a really suitable instrument: the Game Theory.

The Game Theory is a mathematical tool thought by John von Neumann in the 20's of the last century. It arose to the rank of discipline after the von Neumann and Morgenstern's book [10]. At the beginning it was created mainly to analyze economic situations in order to study what would have been the result of an interaction between many users, e.g. the bid for a good, or the battle of prices between two or more industries. Morgenstern and von Neumann dealt with only cooperative games. Afterwards Game Theory developed through the concept of Nash Equilibrium as solution for non-cooperative games defined by the mathematician J.F.Nash. The Nash Equilibrium idea made the whole theory interesting for various applications: mainly economics which is the Game Theory natural context, but also social sciences, politics, evolutionary biology, and in the last decade also wireless communications.

The idea of a distributed management of spectrum holes makes the Game Theory particularly appropriate for this aim. The properness of the Game Theory is because, differently from the decision theory, it deals with several agents interactions instead of considering only one agent. In particular, the main difference with the decision theory is the dependence of the outcome for the interaction under study. In fact, while in the decision theory it is studied a situation where the unique agent directly affects with its choices the outcome, the Game Theory scope is to analyze situations 
with multiple agents where the outcome achieved by one agent (since now referred as player) strictly depends on both its own choices and the other players actions. The Game Theory finds in Cognitive Radio a natural application as modeling tool to analyze how the resources and the channel access are shared between the terminals. Considering the Mitola's original Cognitive Radio tasks, the Game Theory role is to translate the observations, orientations and preferences of the cognitive terminals into decisions. These decisions can be taken by Cognitive Radios opportunistically or in a cooperative way. This consideration marks the difference between application of non-cooperative or cooperative Game Theory approach in a Cognitive Radio based network.

\subsubsection{Basics elements and different types of game}

The application of Game Theory implies the formulation of a mathematical framework since now referred as game. The main elements of a game, independently by its cooperative or non-cooperative nature, are:

- the number of active players looking for radio resources;

- a set of strategies players to choose from;

- a set of payoff functions mapping the vector of strategies chosen by all the players into a preference parameter or number.

In the Cognitive Radio context the players are the cognitive terminals operating within a certain area and in a given frequency bandwidth as secondary users. The set of strategies available to each player is settled by all actions a player can use. In a telecommunications context possible actions are the choices of transmission parameters e.g. modulation, coding rate, transmission power, time slot to occupy, amount of bandwidth to be used. Of course the action to be undertaken can imply the choice of just one or even more parameters. How many quantities are involved in the decision process it is up to the Cognitive Radio network designer; it has to be remembered however that more parameters are considered in the game framework, more complex it is to find a solution. In fact keeping into account more options to choose from, has as consequence a huge increase in the total number of strategies combinations and then in the solution computation times, as well. So a proper Game Theory based decision framework for Cognitive Radio has to be a tradeoff between potential high performances achieved with an accurate model and the implementation feasibility of the same model. As far as it concerns the payoff functions, they translate the vector of the adopted strategies by all players, i.e. a vector of real numbers, into a real value representing the level of satisfaction for the outcome achieved. The key element of the Game Theory emerges right here: a change of strategy by one player affect the real value given by payoff functions of the other players. This aspect makes the payoff functions the decisions engine of the whole framework; indeed for a player, through them, it is possible to analyze the structure of the game evaluating the most convenient strategies given the opponent strategies. 
It is possible to point out several typologies of games, each one with well defined features, [11] and [12]. In the following some of them are described:

- non-cooperative games

- potential games

- repeated games

- cooperative games

- hierarchical games

- stochastic games

Non-cooperative games: they are most used in applications. They are suitable to model interactions having the following features: firstly, each player behaves in a selfish way, assuring the context under study is non-cooperative. Secondly, no agreements or coalitions are possible; in third place each player acts independently, i.e. it is not conditioned or piloted by other players in its decision; the interaction between players happens only once. The solution for non-cooperative games is better known as Nash Equilibrium point [13]. Nash Equilibrium can be described as the point, in terms of vector of strategies, at which no user achieves gain deviating from it. Formally Nash Equilibrium, in terms of payoff value for the $i$-th player, is defined in the following way:

$$
u_{i}\left(s_{i}^{*}, \mathbf{s}_{-\mathbf{i}}^{*}\right) \geq u_{i}\left(s_{i}, \mathbf{s}_{-\mathbf{i}}^{*}\right)
$$

where $u_{i}: R^{N} \rightarrow R$ is the payoff function for the $\mathrm{i}$-th user, $s_{i}$ is the strategy adopted by $\mathrm{i}$-th user, and $\mathbf{s}_{-\mathbf{i}}^{*}$ is the vector of best strategies available for the opponents. Nash Equilibrium requires existence conditions in terms of payoff functions and strategies set shape.

Potential games: they are a different approach to games in strategic form, [15], which is one possible representation for Game Theory frameworks. Potential games are so called because it is possible to define a potential function, valid for all players, mapping a given strategies profile into a unique real number. Furthermore it has been demonstrated the strategies profile maximizing the value given by potential function corresponds exactly with Nash Equilibrium. Then it is opportune to threat a game as a potential game when the formulation allows it, since the advantages can derive from the potential function: it is more practical to deal with a unique function to maximize instead of researching for an equilibrium point satisfying (1.11) into the strategy space.

Repeated games: they are suitable to model situations where the players interact more than once, [11]. Repeated games can treat both finite and infinite number of iterations, with differences in terms of the equilibrium point that is reached. A repeated game is created from a game in strategic form, keeping constant the number of players and the available strategies. As far as it concerns the utility functions since the game is structured in stages there are two possibilities: keeping into account in payoff functions the result achieved in the former stages, or 
forgetting completely what happened before; the latter one is the subclass of myopic repeated games, where players concentrate only on the present situation not considering neither the future nor the past outcomes.

Cooperative games: they are proper to model interactions where the scopes of participants are not the same but they are neither opposed, [14]. Differently from non-cooperative ones, in cooperative games players are able to reach a constraining agreement and respect it. Players can coordinate on the agreement exchanging reciprocal informations. This class of games do not have the Nash Equilibrium as solution, but a point with different characteristics known as Nash Bargaining Point. Differently from the Nash Equilibrium, this new solution point is Pareto-optimal and, with specific payoff functions, it guarantees fairness for the players.

Hierarchical games: they have been thought to analyze situations where there are two classes of player organized hierarchically: manager users and common users, [12]. A common user is typically located in a group of players administrated by a manager. Common users within a group interact between them but not with manager players. Managers can determine the game parameters and rules in their own group and interact with the other managers; moreover common user payoffs are involved in the payoff function of their manager. Then the equilibrium concerns both master and common users, and actually it is not trivial to find a solution for this class of games.

Stochastic games: they represent a more complex Game Theory framework which further encompasses the concept of probability, [16]. That is possible extending the basic elements of a game with two additions: a set of states, which it is the result of the product of states available for each player, and a set of transition function which expresses the probability to pass from one of the available states to another when a certain profile of strategies is played by the players of the game. Stochastic games are actually a generalization of a Markov decision process that can be seen as a stochastic game with only one player.

\subsubsection{Example of application to telecommunications}

It is interesting to point out a possible application of the Game Theory in a Cognitive Radio context. In the following a simple and intuitive example is provided. An FDMA based Cognitive Radio network where two Cognitive terminals are competing for three FDMA slots is considered in Fig. 1.10 and 1.11. The figures represent two possible situations in terms of strategic choices performed by Cognitive Radio terminals: in the first one, Fig.1.10, terminal B occupies two FDMA slot while terminal A is occupying just one slot; in the second figure, Fig. 1.11, both terminals simultaneously access to two FDMA slots, impairing the transmission on the com- 
mon slot. It is worth noting that it has been assumed the terminal A starts the slot occupation from slot number 1 and afterwards it can further occupy the slot number 2 ; differently the terminal B starts the slot occupation from slot number 3 and afterwards it can further occupy slot number 2 . This assumption avoids to complicate the model and it allows to study a reduced number of cases for the game outcome. It is

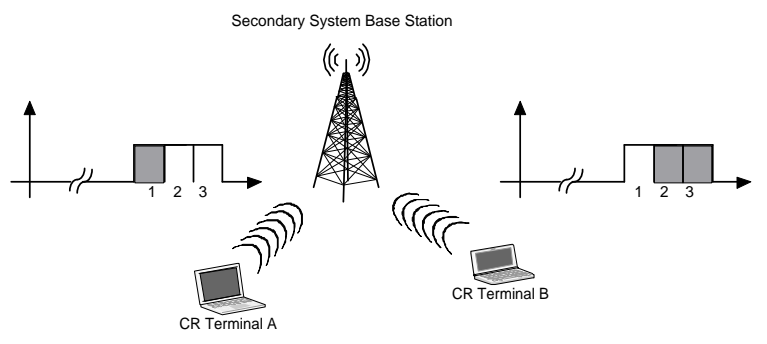

Fig. 1.10: Game Theory application example: Case A

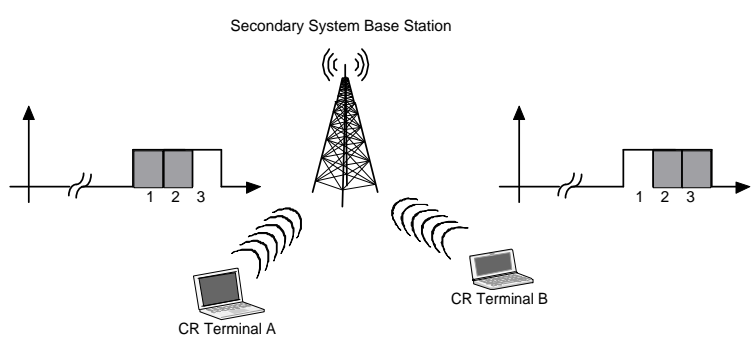

Fig. 1.11: Game Theory application example: Case B

considered that the Cognitive terminals are the players of the game and the number of FDMA slots eligible for occupation are the object of the strategies available to players. It is assumed that there are three adjacent FDMA slots available. The game is simplified assuming the players have only two strategies: to occupy one slot or to occupy two slots. Furthermore it is assumed that the game is played only once and no communication is present. As for the payoff functions, the following three rules to assign revenues to the two players are imposed:

1. a player has a positive gain equal to 3 for each FDMA slot occupied;

2. a player has a penalization equal to 2 for each slot which is also occupied by the other terminal, i.e. the gain is negative;

3. every player has a positive gain equal to 2 for each slot left free.

The fixed payoff values represent actually the logic of the game. In fact, the gain of 3 for each slot achieved gives convenience to players to occupy as much slots 
as possible, and this aspect points out the created game is actually non-cooperative. By the way in order to compensate the impairing tendency to occupy all slots it has been introduced a penalization value of 2 , in case of interference, in order to dissuade this kind of behavior. Furthermore in this model particular importance is given to those outcomes of the game leaving one slot free for contingent other users, and actually this leaves a possible cooperative approach to a game originally thought as non-cooperative. This will be more evident when the possible solutions will be described. In Tab. 1.1, it is shown the tabular representation associated to the FDMA game, given the considered rules. In Tab. 1.1 the rows are the strategies for Cognitive

Table 1.1: Matrix representation of the game for FDMA slots

\begin{tabular}{c|c|c|} 
& $\begin{array}{c}\text { Terminal B } \\
\text { occupies 1 slot }\end{array}$ & $\begin{array}{c}\text { Terminal B } \\
\text { occupies 2 slots }\end{array}$ \\
\hline $\begin{array}{c}\text { Terminal A } \\
\text { occupies 1 slot }\end{array}$ & $(5,5)$ & $(3,6)$ \\
\hline $\begin{array}{c}\text { Terminal A } \\
\text { occupies 2 slots }\end{array}$ & $(6,3)$ & $(4,4)$ \\
\hline
\end{tabular}

Radio terminal A, while the columns are available strategies for CR terminal B. The couples of values within brackets are payoffs values achieved by players: the leftsided one is relative to terminal $\mathrm{A}$, the right-sided one to terminal $\mathrm{B}$. This game is known in literature as prisoner's dilemma and it is has been exhaustively analyzed. According the Nash Equilibrium definition, given by equation (1.11), and payoffs of Tab. 1.1, the equilibrium point of the game is achieved when both users occupy two FDMA slots. This equilibrium point is evidently the sub-optimal one, since the optimal equilibrium point in this case it is achieved when both users choose to occupy only one slot, e.g. payoff achieved by both users equal to 5. Allowing communication and coordination between players, and considering as solution of the game, instead of Nash Equilibrium definition (1.11), the Pareto-optimal point, i.e. the Nash Bargaining point, this can be solved with a cooperative approach. By a practical point of view, this last approach can be addressed as cooperative because it avoids interference between the two players and, more fundamental, it leaves free one FDMA slot perhaps for another incoming terminal.

\subsection{Conclusions and trends}

The ever increasing demand for new and improved services is deeply changing the telecommunication industry. Radio access is no longer considered as the bottleneck of the information delivery chain, but a new opportunity for mobile broadband services. In this framework, the efficient use of spectrum resources plays a fundamental role in the deployment of future generation networks and in the fulfillment of the 
globalization process. A new gold rush is begun. But flexibility in spectrum usage is not only an "engineering" issue: it also represents a great social and economical opportunity for emerging countries. Universal radio devices have the potentials of the Global Market, with a compact (but smart) production load. This aspect can be successfully exploited by emerging economies willing to gain a position in the world communication industry.

The dynamic and flexible usage of the radio spectrum is the key enabling technology for the universal abstraction of the network access. The connection between communication services and their transport mechanism is finally broken, allowing users to use their services without concerns about geographical position, motion, situation and device capability. The "perfect" communication service is only a few years apart.

Cognitive radio is the most effective solution to the widespread waste of radio resources due to the poor utilization of large licensed bands. Digital Television, Video compression and Dynamic Resource Management tends to compact licensed spectrum creating the basis for this new paradigm of radio access. Cognitive radio has two main approaches: heuristic and gaming. The heuristic method allows devices in a clear and defined context to use efficiently free spectrum holes, without interfering with the licensed communication service. Examples in both satellite and terrestrial scenarios have been provided. Game theory gives another perspective to CR systems. In games, players subject to a set of rules can take decisions even if they are in complex situations. When applied to CR, game theory can solve complex coexistence scenarios with distributed decisions, actually inserting some intelligent functions into devices. A game theoretical approach to CR is described, showing basic principles and specific proposals from the scientific community.

Concluding, a new telecommunication era is approaching, being characterized by a large platform new services and a powerful convergence of access technologies into a unified universal network. A flexible use of spectrum represents the required milestone in this evolution and the CR can be the key concept to carry it out.

\section{References}

1. O. Ileri O. and N. B.Mandayam, Dynamic spectrum access models: toward an engineering perspective in the spectrum debate, IEEE Communications Magazine, vol 46, Issue 1, pp. 153-160, January 2008

2. M. McHenry and D. McCloskey, New York City Spectrum Occupancy Measurements, September 2004, December 2004.

3. Convergence and Next Generation Networks, OECD Ministerial meeting, 17-18 June 2008, Seoul, Korea, http://www.oecd.org/dataoecd/25/11/40761101.pdf

4. J. Brito, The Spectrum Commons in Theory and Practice, working paper in regulatory studies, 2006.

5. J. M. Peha, Approaches to Spectrum Sharing, IEEE Communications Magazine, vol. 43, no. 2, pp. 10-12, February 2005.

6. VICOM (Virtual Immersive COMmunications) project, http://www.vicom-project.it

7. J. Mitola, "Cognitive Radio: Making software radios more personal". IEEE Pers. Commun., August 1999 
8. FCC Spectrum Policy Task Force, "FCC Report of the Spectrum Efficiency Working Group", Nov. 2002, http://www.fcc.gov/sptf/files/SEWGFinalReport1.pdf/

9. Cognitive Radio Technology, Editor Bruce Fette, Newnes, 2006

10. J. von Neumann and O. Morgenstern, Theory of Games and Economic Behavior, Princeton University Press, Princeton, 1947

11. D. Fudenberg and J. Tirole,Game Theory, The MIT Press, Cambridge, 2001

12. T. Basar and G. J. Olsder, Dynamic Noncooperative Game Theory, 2nd Ed., SIAM, Philadelphia, 1999

13. J. F. Nash, "Non-cooperative games", The Annals of Mathematics, vol. 54, no. 2, pp. 286295,1951

14. J. F. Nash, "Two person cooperative games", Econometrica, vol. 21, no. 1, pp. 128-140, 1953

15. D. Monderer and L. S. Shapley, "Potential games", Games and Economic Behavior, vol. 14, no. 1, pp. 124-143, 1996

16. L. S. Shapley, "Stochastic games", Proceedings of the National Academy of Sciences, vol. 39, pp. 1095-1100, 1953

17. ETSI EN 302 583, v. 1.1.1, "Digital Video Broadcasting (DVB); Framing structure, channel coding and modulation for Satellite Services to handheld devices (SH) below 3Ghz”, 200803 .

18. E. Lutz,Daniel Cygan, Michael Dippold, Frank Dolainsky, and Wolfgang Papke "The Land Mobile Satellite Communication Channel-Recording, Statistics, and Channel Model", IEEE Transaction on Vehicular Technology, vol.40, No.2, May 1991 